\title{
Power-Matching Based SOC Balancing Method for Cascaded H-Bridge Multilevel Inverter
}

\author{
Jingrong Yu, Gang Zhang, Mingkai Peng, Dongran Song, and Maoyun Liu
}

\begin{abstract}
A power-matching based balancing method is proposed to solve the unbalanced state of charge (SOC) of the DC-side batteries in cascaded H-bridge multilevel inverter (CHBMI). First, the essential reason for SOC imbalance in CHBMI with phase disposition (PD) pluse width modulation (PWM) is analyzed, and the disadvantages of the voltage-matching based SOC balancing method are pointed out. Then, from the perspective of powermatching, an SOC balancing method is proposed to achieve fast SOC balance under arbitrary load characteristics. In addition, a power-matching algorithm avoiding zero-crossing detection is presented, which brings about accuracy and simplicity of the proposed method. Finally, the parameter of the proposed method is tuned by taking the switching loss and balancing speed into consideration. The simulation and experimental results show that the proposed method is of lower switching loss and faster balancing rate under arbitrary load characteristics.
\end{abstract}

Index Terms-Multilevel converter, phase disposition (PD)-pluse width modulation (PWM), power-matching, state of charge (SOC) balancing.

\section{INTRODUCTION}

C ASCADED H-bridge multilevel inverter (CHBMI) constructed by series-connected H-bridges, is a popular multilevel topology featuring good redundancy, low switching frequency, low loss, and less output harmonics. Compared with the diode-clamped [1]-[3] and flying capacitor multilevel topologies [4]-[5], the topology of CHBMI is more suitable for the systems supplied by multiple independent DC sources. Because most DC sources are low voltage, the large number of series connected DC sources can be avoided in CHBMI [6][8]. Hence, CHBMI-based energy systems, comprehending low voltage $\mathrm{DC}$ sources and outputting high $\mathrm{AC}$ voltage, such as energy storage systems (ESSs), super capacitors systems, high-voltage direct current (HVDC) and electrical motors, have been widely used in engineering [9]-[10]. In CHBMIbased systems with batteries or super capacitors as the DC sources, the state of charge (SOC) imbalance of batteries or super capacitors may occur because of the different initials, the different characteristics of DC sources such as internal

Manuscript received July 8, 2020; accepted September 14, 2020. Date of publication December 30, 2020; date of current version December 8, 2020.

All authors are with the School of Automation, Central South University, Changsha 410083, China (e-mail: Jingrong_yu@126.com; 2632880842@ qq.com; 584752016@qq.com; humble_szy@163.com; liumaoyun321@126. com).

Digital Object Identifier 10.24295/CPSSTPEA.2020.00029 impedance, self-discharge rate, and the different modulation techniques of the inverters such as phase shift (PS) and phase disposition (PD) pluse width modulation (PWM) [11]-[12]. Since the SOC imbalance issues will reduce the reliability and lifetime of the whole system, many balancing methods have been explored for CHBMI-based systems.

Among the SOC balancing methods, there is a special one called power balancing method, which deals with SOC imbalance caused by modulation [13], and achieves power balance between modules with modified modulation technique. PS and PD PWM are the commonly used modulation techniques to achieve power balancing, of which PS-PWM can naturally balance the output power, but PD-PWM requires extra enhancement. Hence, a simple and effective power balancing method based on modified PD-PWM is proposed in [14], which rotates the carrier signal of modules in each switching cycle. While this power balancing method is only suitable for low modulation ratio due to its complexity. Reference [15] proposes a method that interchanges the voltage waveform of each module in each $1 / 4$ fundamental period and realizes power balancing in one fundamental period. However, when the number of modules is larger than 2, the output power of modules may be roughly balanced. In [16], an approach is proposed to deal with the power imbalance problem in every $3 / 4$ fundamental period of output voltage, but the carriers need to be rearranged as the number of the modules increases. It should be noted that all power balancing methods are unable to deal the SOC imbalance in case of different SOC initial values and different charging or discharging characteristics.

While the SOC balancing methods can deal with the issues caused by the different initials, interior impedance and discharging rates of DC sources in CHBMI-based systems, they are more advantageous and more useful in engineering. Some active SOC balancing circuits have been proposed, in which additional circuits are employed. In reference [17], an active SOC balancing control strategy for modular super capacitor ESS is proposed, which requires a master-slave structure including a common DC bus voltage loop and individual slave current loop for each sub-module. However, extra control circuits increase the system complexity and reduce system efficiency. Considering that a practical application requires fast balancing during transient disturbances, a balancing control strategy with extra degree of freedoms and independent power control capability is proposed in [18]-[19], in which a resonant circuit is added in the topology of CHBMI to realize SOC balancing by enabling the 
power exchange among modules under PS-PWM. Although the SOC balancing process only requires one extra resonant circuit, the control complexity is still challenging.

Consequently, some passive SOC balancing methods requiring no additional active circuits have been proposed, which match the current SOC values with the output voltages of the modules, and allocate the working state of the modules according to the matching results. Since the matching between SOC and voltage is intuitive from the perspective of voltagematching, various SOC balancing methods with different modulation techniques have been explored. An SOC balancing method based on PS modulation is proposed in [20], which modifies the control signal in each duty cycle according to the energy balance control, while the voltage distortion is relatively serious. In references [21] and [22], a balancing method based on staircase modulation is proposed with switching sequence rotation and sorting redundancy selection approaches. However, when the number of modules is small, the switching harmonics of output voltage are large. Reference [23] proposes a PD-PWM based selective loop bias mapping (LBM) method, in which the min and max modules SOC and their index are directly selected, and the rest of modules follow the LBM rule that will achieve power balancing. While only two modules in this method achieve SOC balancing in each switching cycle, which will decrease the speed of SOC balancing in highvoltage applications with more modules and cause unnecessary switching during balancing. In reference [24], a fundamental frequency sorting strategy with PD-PWM is proposed. When there is a phase difference between voltage and current, the fundamental frequency sorting cannot achieve a fast and good balancing consistency.

With the above analysis, it can be known that passive SOC balancing methods are more favorable for their simplicity, of which the PD-PWM based balancing method with higher degree of freedom in utilizing redundant switching patterns, features good performance in voltage quality and balancing speed. However, the existing PD-PWM based balancing methods are all based on voltage-matching rule. When there is phase difference of load impedance, zero detection circuit or algorithm must be required, which will bring about detection errors and increase system complexity.

To solve the issue, an SOC balancing method based on power-matching is proposed in this study. By analyzing the relationship between the discharge capacity of carriers and the DC side SOC of batteries, the issue of the voltage-matching based methods is pointed out, and the issue is solved from the perspective of power-matching. With the proposed method, the SOC balancing speed under arbitrary load characteristics can be attained without zero crossing detection. An approach of instantaneous power calculation in each switching cycle is presented to determine the sorting change in the fundamental period. The relationship between key parameters and performance of SOC balancing method based on powermatching is analyzed, which provides theoretical basis for the parameter design of the proposed SOC balancing method.

The rest of the paper is organized as follows: The principle

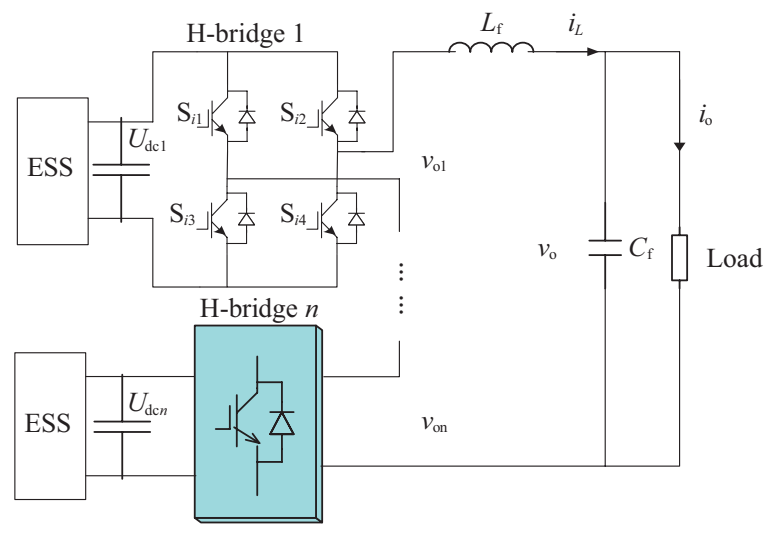

Fig. 1. The topology of cascade H-bridge multilevel inverter.

TABLE I

Switching Mode of Modules

\begin{tabular}{lcccc}
\hline \hline Output voltage & $\mathrm{S}_{i 1}$ & $\mathrm{~S}_{i 2}$ & $\mathrm{~S}_{i 3}$ & $\mathrm{~S}_{i 4}$ \\
\hline$+U_{\mathrm{dc}}$ & 1 & 0 & 0 & 1 \\
0 & 1 & 1 & 0 & 0 \\
0 & 0 & 0 & 1 & 1 \\
$-U_{\mathrm{dc}}$ & 0 & 1 & 1 & 0 \\
\hline \hline
\end{tabular}

of CHBMI and the voltage-matching based balancing method are given in Section II. The proposed method and performance analysis are given in Section III. Parameter tuning of the proposed method is given in Section IV. The results of simulation and experiment are demonstrated in Section V. Lastly, Section VI summarizes the paper.

\section{Principle of CHBMI and the Voltage-Matching Based SOC Balancing Method}

In this section, the principle of CHBMI is presented, and the limitations of voltage-matching based SOC balancing method are discussed.

\section{A. Operating Principle of CHBMI}

The configuration of CHBMI is shown in Fig. 1. The CHBMI includes $n$ series-connected H-bridges and $n$ energy storage components whose outputs are series connected to the load. An output filter consisting of $L_{\mathrm{r}}$ and $C_{\mathrm{r}}$ is paralleled with the cascaded H-bridges.

According to the instantaneous value of voltage reference in each switching cycle, the PD-PWM assigns appropriate switching modes to each cell in a way that the CHBMI synthesizes voltage reference at its AC terminals. Possible switching modes for $i^{\text {th }}$ cell and their corresponding output AC voltages are defined in Table I.

The mathematical model of single-phase voltage PWM inverter can be expressed as (1)-(3) with state-space average method. 


$$
\begin{gathered}
C \frac{\mathrm{d} v_{\mathrm{o}}}{\mathrm{d} t}=i_{L}-i_{\mathrm{o}} \\
L \frac{\mathrm{d} i_{L}}{\mathrm{~d} t}=v_{\mathrm{sum}}-v_{\mathrm{o}}=\left(v_{\mathrm{o} 1}+v_{\mathrm{o} 2}+\ldots+v_{\mathrm{on}}\right)-v_{\mathrm{o}} \\
L \frac{\mathrm{d} i_{L}}{\mathrm{~d} t}=\left(v_{\mathrm{o} 1}+v_{\mathrm{o} 2}+\ldots+v_{\mathrm{o} n}\right)-v_{\mathrm{o}} \\
=d_{1} U_{\mathrm{dc} 1}+d_{2} U_{\mathrm{dc} 2}+\ldots+d_{n} U_{\mathrm{dc} n}-v_{\mathrm{o}}
\end{gathered}
$$

Where $U_{\mathrm{dc}}$ is defined as the voltage of DC side, $v_{0}$ is the voltage on the capacitor, $i_{L}$ is inductive current, and $i_{0}$ is load current.

Supposing that $u_{\mathrm{ref}}$ represents the reference voltage of the inverter and $U_{\text {ref }}$ is the amplitude, $n$ represents the total number of modules, $U_{\mathrm{dc} 1}, U_{\mathrm{dc} 2}, \ldots, U_{\mathrm{dc} n}$ represents the DC side voltage of modules, $T_{\mathrm{s}}$ represents switching cycle; $T_{1}, T_{2}, \ldots, T_{n}$ is the running time of each H-bridge module within a switching cycle, the total output voltage of the inverter is:

$$
u_{\text {ref }}=U_{\text {ref }} \sin \omega t
$$

The reference voltage can be approximated to a constant value in one switching cycle:

$$
\left\{\begin{array}{l}
u_{\mathrm{ref}} \cdot T_{\mathrm{s}}=U_{\mathrm{dcl}} \cdot T_{1}+U_{\mathrm{dcc}} \cdot T_{2}+\ldots+U_{\mathrm{dcn}} \cdot T_{n}, u_{\mathrm{ref}}>0 \\
u_{\mathrm{ref}} \cdot T_{\mathrm{s}}=-U_{\mathrm{dcl}} \cdot T_{1}-U_{\mathrm{dc} 2} \cdot T_{2}-\ldots-U_{\mathrm{dcn}} \cdot T_{n}, u_{\mathrm{ref}}<0
\end{array}\right.
$$

When the DC side voltage of each module is equal $\left(U_{\mathrm{dcl}}=\right.$ $\left.U_{\mathrm{dc} 2}=, \ldots,=U_{\mathrm{dc} n}=U_{\mathrm{dc}}\right),(5)$ can be obtained:

$$
\left\{\begin{array}{l}
\frac{u_{\mathrm{ref}}}{U_{\mathrm{dc}}}=\frac{T_{1}+T_{2}+\ldots+T_{n}}{T_{\mathrm{s}}}, u_{\mathrm{ref}}>0 \\
-\frac{u_{\mathrm{ref}}}{U_{\mathrm{dc}}}=\frac{T_{1}+T_{2}+\ldots+T_{n}}{T_{\mathrm{s}}}, u_{\mathrm{ref}}<0
\end{array}\right.
$$

The region $k$ of $u_{\text {ref }}$ and the average voltage $v_{\mathrm{c} i}$ of the $i^{\text {th }}$ carrier can be calculated as follows:

$$
\begin{gathered}
k=\text { floor }\left[\operatorname{abs}\left(\frac{u_{\mathrm{ref}}}{U_{\mathrm{dc}}}\right)\right] \\
\left\{\begin{array}{l}
T_{1}=T_{2}=\ldots=T_{n-k-1}=0 \\
T_{n-k}=\left(\frac{u_{\mathrm{ref}}}{U_{\mathrm{dc}}}-k\right) \cdot T_{\mathrm{s}} \\
T_{n-k+1}=T_{n-k+2}=\ldots=T_{n}=T_{\mathrm{s}} \\
v_{c i}=U_{\mathrm{dc}} \cdot T_{i} / T_{\mathrm{s}}
\end{array}\right.
\end{gathered}
$$

From (6)-(8), when $u_{\mathrm{ref}}>0, z$ modules need to work in the " 1 " state, only one module works in the "PWM" state, and $n-z-1$ modules work in the " 0 " state. Similarly, when $u_{\text {ref }}<0, z$ modules work in the "- 1 " state, one module works in the "PWM" state, and $n-z-1$ modules work in the " 0 " state.

\section{B. Limitation Analysis of Voltage-Matching Based Method}

PD-PWM is widely used in CHBMI. However, due to the modulation or differences between the electrochemical cells,

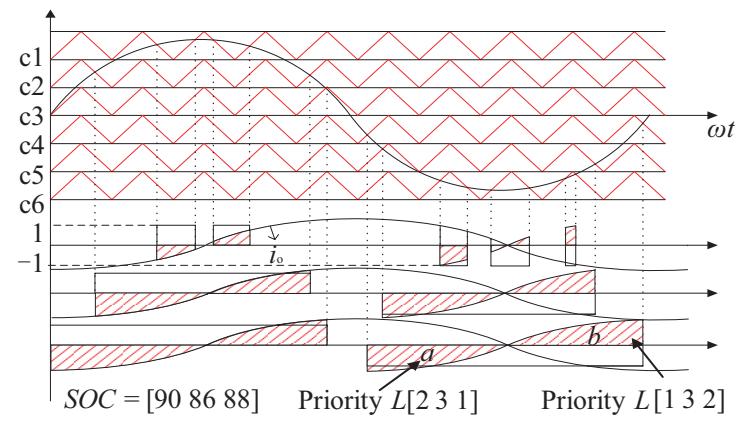

Fig. 2. Voltage matching based SOC balancing.

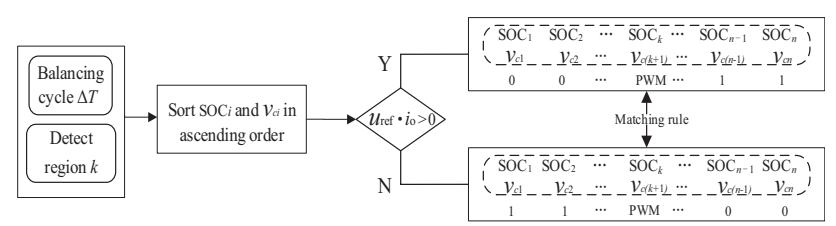

Fig. 3. Diagram of the voltage-matching considering current direction.

the modules may have different duty ratio. Inevitably, some modules may work in high duty ratio situation, which will increase switching loss and power consumption of modules. Thus, the SOC of the battery will be unbalanced. The voltage of battery will drop, which reduces the lifetime of DC source. According to (3), because of the voltage drop, the gain of the control transfer function will decrease, and the actual output voltage of the inverter will be lower than the reference value, which has side effect on the stability of inverter.

Fig. 2 shows PD modulation which results in each module experiencing different duty cycles. A balancing strategy is presented, in which the above property of the multilevel converter is used in order to enforce different modules currents by controlling the position of each module in the priority list $L$, referred to as module position. Supposing that SOC of each module is [90 86 88], the priority list would be $\left[\begin{array}{ll}1 & 3\end{array}\right]$. The voltage-matching rule assigns modules with different SOC mapping to the carrier with different discharge capacity according to the priority list. That is to say, the module corresponding to the max of SOC will work in the carrier with strong discharge capability.

It should be noted that when the load is inductive load or capacitive load, since there is phase difference between the output voltage and the output current (the maximum phase difference is 90 degrees). Under this situation, the output power is negative in area $a$. Therefore, the matching according to the output voltage may fail when system is running from area $a$ to area $b$, which will result in slower SOC balance speed of the battery. To solve the above issue, one solution is to reverse the priority list when the direction of current changes. For example, the priority list is [ [ $\left.\begin{array}{lll}2 & 3 & 1\end{array}\right]$ in area $a$. In area $b$, the priority needs to turn into [lll $\left.\begin{array}{ll}1 & 3\end{array}\right]$. The improved voltagematching based SOC balancing method considering the current direction is demonstrated in Fig. 3.

In Fig. 3, $\Delta T$ is used as the balancing cycle for SOC balancing method, which can be designed larger than the 


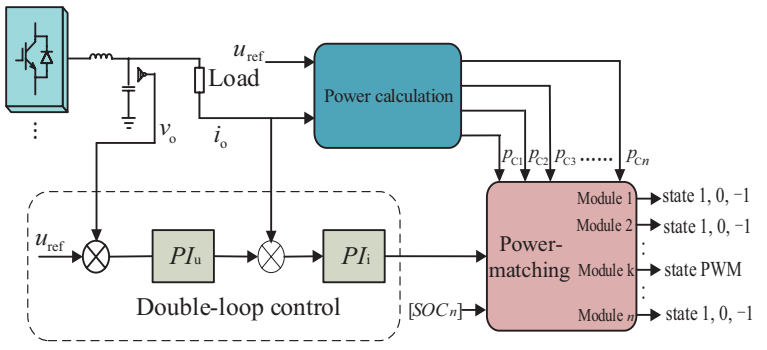

Fig. 4. Control system of single-phase inverter with power-matching based SOC balancing method.

switching cycle $T_{\mathrm{s}}$ to avoid frequent sorting and carrier selection. By calculating the location region $k$ of the reference voltage and the voltage of each module, the output voltage $v_{\mathrm{c}_{i}}$ and $S O C_{i}$ for all modules can be sorted, and the SOC balancing can be achieved by voltage-matching. It can be seen from Fig. 3 that zero detection is required to employ different matching rules, which will bring about detection errors and increase the system complexity.

\section{The SOC Balancing Method Based on Power-Matching}

To address the issue of voltage-matching, a novel SOC balancing method is proposed from the perspective of powermatching, in which a power calculation approach is presented to provide accurate instantaneous power values of each module, and a power-matching approach is employed to achieve SOC balancing with fast balancing speed and no zerodetection.

\section{A. Framework of the Proposed SOC Balancing Method}

The diagram of the SOC balancing method based on powermatching is illustrated in Fig. 4, which consists of voltage and current double-loop control, the power calculation unit and the power-matching unit.

In Fig. 4, the voltage and current double-loop control is the classical one for single-phase inverters. In outer loop of voltage control, the output voltage $v_{\mathrm{o}}$ is compared with its reference signal $u_{\text {ref }}$, and the error signal between the two is processed by the voltage controller $P I_{\mathrm{u}}$ to obtain the reference for the current loop. In the inner current loop, the output of voltage controller is compared with output current $i_{0}$, and the error signal is processed by the inner current controller $P I_{\mathrm{i}}$ to give the modulation wave for PD-PWM.

Apart from the control part, the SOC balancing method is supplemented in Fig. 4. Firstly, power calculation is responsible for calculating real-time power of each module, of which the instantaneous power $\left(p_{\mathrm{cl}}, \ldots, p_{\mathrm{c} n}\right)$ of the voltage and current is calculated at frequency $f_{\mathrm{s}}$. Then, the SOCs $\left(S O C_{1}, \ldots, S O C_{n}\right)$ of all the modules are sampled. In each balancing cycle $\Delta T$, the instantaneous power $\left(p_{\mathrm{cl}}, \ldots, p_{\mathrm{c} n}\right)$ and the SOCs $\left(S O C_{1}, \ldots\right.$, $S O C_{n}$ ) are sorted and matched. Lastly, according to the powermatching sequence, the modulation signals will be distributed to each module to achieve SOC balance.

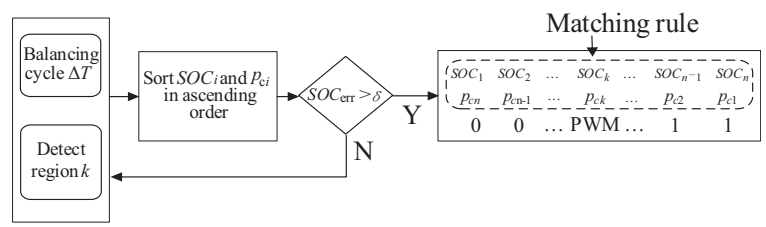

Fig. 5. The diagram of the power-matching.

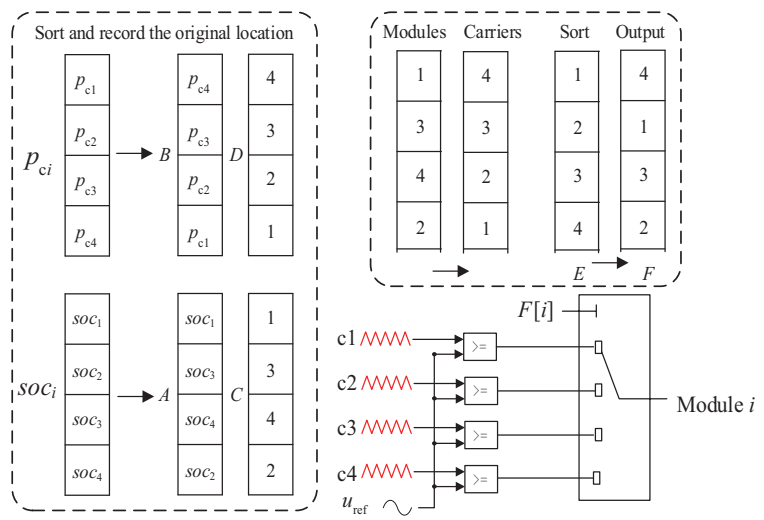

Fig. 6. The matching rule for power and SOC.

\section{B. The Power-Matching Algorithm}

Fig. 5 shows the diagram of power-matching based SOC balancing method. The SOC and power of each module are sorted in ascending order.

Supposing that $S O C_{\text {err }}$ is the maximum SOC difference, it can be defined as:

$$
\left\{\begin{array}{l}
\overline{S O C}=\frac{1}{n} \sum_{i=1}^{n} S O C_{i} \\
S O C_{\mathrm{err}}=\max \left|S O C_{i}-\overline{S O C}\right|, i=1,2, \ldots, n
\end{array}\right.
$$

If the $S O C_{\text {err }}$ is greater than the given error coefficient $\delta$, the power-matching is updated; otherwise, the original matching is maintained.

According to Figs. 3 and 5, it can be seen that with the powermatching, the balancing method does not need additional zerocrossing detection circuit.

The matching rule in the proposed method is developed by selecting modules with different SOC corresponding to different discharge capacity of the carrier. Taking the nine levels inverter with four modules for example, the matching process is shown in Fig. 6.

The workflow of the matching algorithm is as follows:

Step 1: sort array $S O C_{i}$ and array $p_{\mathrm{c} i}$ to get array $A$ and array $B$, record the original position of each element after sorting and generate array $C$ and array $D$;

Step 2: establish the one to one correspondence between array $C$ and array $D$;

Step 3: sort array $C$ to get array $E$ and $F$;

Step 4: output array $F$.

Hence, the matching algorithm is carried out in each balancing cycle $\Delta T$ with the sorting results of the instantaneous power 
$\left(p_{\mathrm{cl}}, \ldots, p_{\mathrm{cn} n}\right)$ and the SOCs $\left(S O C_{1}, \ldots, S O C_{n}\right)$.

\section{The Power Calculation Approach}

It should be noted that the balancing cycle $\Delta T$ is designed by making a trade-off between balancing speed and switching loss, thus it may be variable in different cases, and this point will be discussed in next section.

To provide accurate power values, instantaneous power of each module is employed for power-matching, and the derivation of power calculation of each module is presented here.

According to the analysis of voltage in (4), the power equation can be obtained as (11):

$$
u_{\mathrm{ref}} \cdot i_{\mathrm{o}} \cdot T_{\mathrm{s}}=U_{\mathrm{dc} 1} \cdot i_{\mathrm{o}} \cdot T_{1}+U_{\mathrm{dc} 2} \cdot i_{\mathrm{o}} \cdot T_{2}+\ldots+U_{\mathrm{dc} n} \cdot i_{\mathrm{o}} \cdot T_{n}
$$

Integrate both sides of (11):

$$
T_{\mathrm{s}} \int_{t}^{t+T_{\mathrm{s}}} u_{\mathrm{ref}} \cdot i_{\mathrm{o}} \mathrm{d} t=T_{1} \int_{t}^{t+T_{\mathrm{s}}} U_{\mathrm{dc} 1} \cdot i_{\mathrm{o}} \mathrm{d} t+\ldots+T_{n} \int_{t}^{t+T_{\mathrm{s}}} U_{\mathrm{dcn}} \cdot i_{\mathrm{o}} \mathrm{d} t
$$

Dividing both sides of (12) by $T_{\mathrm{s}}$, (13) can be derived as:

$$
p_{\text {sum }}=T_{1} p_{\mathrm{c} 1}+T_{2} p_{\mathrm{c} 2}+\ldots+T_{n} p_{\mathrm{c} n}
$$

Where $p_{\text {sum }}$ is the total output power of the inverter, $p_{\mathrm{cl}}, p_{\mathrm{c} 2}$, $\ldots, p_{\mathrm{c} n}$ is the output power of each carrier. Assuming the $u_{\mathrm{ref}}$ and $i_{\mathrm{o}}$ remain constant within one $T_{\mathrm{s}}$, according to (6), (7), (9), (11) and (12), It is known that the power of each module in $T_{\mathrm{s}}$ is:

$$
\left\{\begin{array}{l}
p_{\mathrm{c} 1}=p_{\mathrm{c} 2}=\ldots=p_{\mathrm{c}(n-k-1)}=0 \\
p_{\mathrm{c}(n-k)}=U_{\mathrm{dc}} \cdot i_{\mathrm{o}} \cdot\left(\left|\frac{u_{\mathrm{ref}}}{U_{\mathrm{dc}}}\right|-k\right) \\
p_{\mathrm{c}(n-k+1)}=p_{\mathrm{c}(n-k+3)}=\ldots=p_{\mathrm{c} n}=U_{\mathrm{dc}} \cdot i_{\mathrm{o}}
\end{array}\right.
$$

According to (5) and (14), the instantaneous power for each module can be calculated with the value and the location region number $k$ of the reference voltage.

\section{Parameter Tuning of the Proposed Method}

In the digital implementation of the proposed SOC balancing method, the performance of switching loss and SOC balancing speed should both be considered. For example, when the SOC balancing cycle $\Delta T$ is equal to the switching cycle $T_{\mathrm{s}}$, better SOC speed can be guaranteed, but higher frequency of power sorting and carrier selection will increase the computational burden and the switching loss of the inverter. Hence, switching loss and balancing speed are discussed here, and the parameter of the proposed method is designed by making trade-off between the two aspects.

\section{A. Switching Loss Analysis}

In this paper, the switching loss without SOC balancing is called necessary switching loss. While switching loss for SOC balancing is called additional switching loss. The additional switching loss is discussed here.

In order to determine the relationship between SOC balancing cycle $\Delta T$ and switching loss, SOC is estimated by:

$$
S O C=S O C_{t=0}-\frac{1}{C_{\mathrm{e}}} \int_{0}^{t} \eta i_{\mathrm{dc}} \mathrm{d} t
$$

Where $S O C_{t=0}$ is the initial value of the SOC, $C_{\mathrm{e}}$ is the battery capacity, and $\eta$ is the battery charging and discharging efficiency. By setting $\eta$ to 1 , according to (15), the SOC variation within an SOC balancing cycle $\Delta T$ can be summarized as follows:

$$
\Delta S O C=\frac{1}{C_{\mathrm{e}}} \int_{0}^{\Delta T}\left|i_{\mathrm{dc}}\right| \mathrm{d} t
$$

The total switching actions of all modules in a fundamental wave cycle $T$ can be expressed as:

$$
n_{\text {total }}=\frac{1}{C_{\mathrm{e}} \Delta S O C} \int_{0}^{T} n_{\text {add }}\left|i_{\mathrm{dc}}\right| \mathrm{d} t+n_{\text {need }}
$$

where $n_{\text {add }}$ is the number of additional switch actions and $n_{\text {need }}$ is the number of necessary switch actions.

According to the PD principle illustrated in Fig. 2, the minimum of $n_{\text {add }}$ is zero, and the maximum is related to the value of the modulated wave, which can be expressed as

$$
n_{\text {add }}=\left\{\begin{array}{l}
2 k, k<\frac{n}{2} \\
2(n-k), k>\frac{n}{2}
\end{array}\right.
$$

From (17) and (18), the larger the $\triangle S O C$ is, the smaller the total switching frequency of modules in a fundamental frequency cycle will be. If $\triangle S O C$ is not controlled, the total switching actions of all modules is equal to the necessary switch actions in a fundamental period. While according to (16), the larger $\triangle T$ is, the larger $\triangle S O C$ will be. That means, the larger $\Delta T$ is, the smaller the additional switching loss is.

Therefore, the value of $\Delta T$ can be appropriately increased to reduce the switching loss. Meanwhile, the large value of $\Delta T$ will decrease the computational burden.

\section{B. Balancing Speed Analysis}

The SOC balancing speed can be evaluated by the output power of each module in a period of given time. Here, the output power in half fundamental period of modules is used to describe the SOC balancing speed.

In order to intuitively illustrate the output power of each module in half fundamental period, the dual Fourier transformation is introduced.

As to PD-PWM, there are $n^{-1}$ modules under the over modulation condition in the case of $n$ modules, and the total equivalent voltage of $i(i=1,2, \ldots, n-1)$ modules can be expressed as: 


$$
\left\{\begin{array}{l}
v_{i}(t)=\frac{n U_{\mathrm{dc}}}{\pi}\left\{M_{i}\left[2 A_{i}-\sin \left(2 A_{i}\right)\right]+4 \cos A_{i}\right\} \sin \omega t \\
A_{i}=\frac{\pi}{2}, M_{i} \leqslant 1 \\
A_{i}=\arcsin \frac{1}{M_{i}}, M_{i}>1
\end{array}\right.
$$

where $M_{i}$ is defined as

$$
M_{i}=\frac{n}{n+1-i} \cdot m
$$

By Substituting (20) into (19), the amplitude $V_{i}$ of $v_{i}(t)$ is:

$$
V_{i}=\frac{1}{\pi}\left(2 \arcsin \frac{1}{M_{i}}+\frac{2}{M_{i}} \sqrt{\frac{M_{i}^{2}-1}{M_{i}^{2}}}\right) \cdot U_{\text {ref }}=H_{i} \cdot U_{\text {ref }}
$$

where $H_{i}$ is the proportionality coefficient.

According to PD-PWM principle, the amplitude of output voltage for each module is:

$$
\left\{\begin{array}{l}
V_{\mathrm{c} 1}=U_{\mathrm{ref}}-V_{2} \\
\vdots \\
V_{\mathrm{c}(n-1)}=V_{n-1}-V_{n} \\
V_{\mathrm{c} n}=V_{n}
\end{array}\right.
$$

The total average power of CHBMI in half fundamental period is:

$$
p_{\mathrm{o}}=\frac{2}{T} \int_{0}^{\frac{T}{2}} U_{\text {ref }} \sin \omega t \cdot I \sin (\omega t-\varphi) \mathrm{d} t
$$

According to (21), (22) and (23), the output power $p_{\text {ci }}$ of the $i^{\text {th }}$ module can be derived:

$$
\left\{\begin{array}{l}
p_{c i}=\frac{2}{T} \int_{0}^{\frac{T}{2}} V_{c i} \sin \omega t \cdot I \sin (\omega t-\varphi) \mathrm{d} t=\left(H_{i}-H_{i+1}\right) \cdot p_{\mathrm{o}} \\
p_{\mathrm{c} 1}=\left(1-H_{2}\right) \cdot p_{\mathrm{o}} \\
p_{\mathrm{c} n}=H_{n} \cdot p_{\mathrm{o}}
\end{array}\right.
$$

where $\varphi$ is the impedance angle of the load.

It can be known from (24) that the output power of each module is proportional to the total power in half fundamental period.

The maximum difference of the output power among modules can be employed to describe the SOC balancing speed. For PDPWM, the power difference between the first module and the last one is the greatest, thus it is used to judge the balancing speed. According to (24), the maximum difference of the output power is written as (25).

$$
p_{\mathrm{cn}}-p_{\mathrm{c} 1}=\left(H_{n}-1+H_{2}\right) \cdot p_{\mathrm{o}}
$$

According to (25), the relationship of the SOC balancing

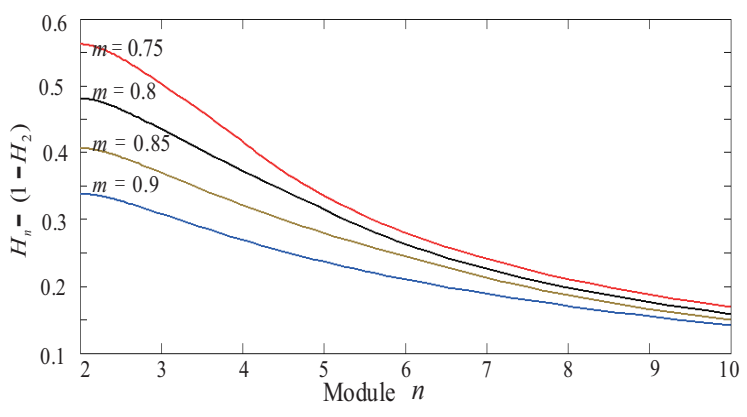

Fig. 7. The relationship between the number of modules and the unbalance degree under different modulation ratios.

speed, the number of modules, and the modulation ratio $m$ are shown in Fig. 7.

From Fig. 7, when $n$ increases, the balancing speed will decrease at the same modulation ratio. On the other hand, the unbalance degree increases with the decrease of modulation ratio at the same number of modules.

For the proposed method, when $\Delta T=0.5 T$, the powermatching of the proposed method cannot change the output power of each module in $0.5 T$. While $\Delta T<0.5 T$, powermatching will change the output power of each module, thus the power difference defined in (26) becomes:

Inductive load:

$$
\begin{aligned}
p_{\mathrm{cn}}-p_{\mathrm{c} 1}= & \left(H_{n}-1+H_{2}\right)\left\{p_{\mathrm{o}}-\left[\frac{1}{T \cdot H_{n}}+\frac{1}{T \cdot\left(1-H_{2}\right)}\right] \cdot\right. \\
& \int_{0}^{\frac{\varphi}{\omega}} U_{\text {ref }} \bullet[[\cos \varphi-\cos (2 \omega t-\varphi)] \mathrm{d} t\}
\end{aligned}
$$

Capacitive load:

$$
\begin{aligned}
p_{\mathrm{cn}}-p_{\mathrm{c} 1}= & \left(H_{n}-1+H_{2}\right)\left\{p_{\mathrm{o}}-\left[\frac{1}{T \cdot H_{n}}+\frac{1}{T \cdot\left(1-H_{2}\right)}\right] \cdot\right. \\
& \left.\int_{\frac{T}{2}-\frac{\varphi}{\omega}}^{\frac{T}{2}} U_{\mathrm{ref}} \cdot I[\cos \varphi-\cos (2 \omega t+\varphi)] \mathrm{d} t\right\}
\end{aligned}
$$

By comparing (26) with (25) and (27) with (25), it can be seen that the power difference increases when using the proposed method.

\section{Tuning of the $\Delta T$}

From the above analysis, it is known that the balancing cycle $\Delta T$ has effect on the switching loss and the balancing speed. So, the tuning of $\Delta T$ is very important.

According to (26) and (27), the maximum of $\Delta T$ is $0.5 T$. While in the digital implementation, the minimum of $\Delta T$ is the switching cycle $T_{\mathrm{s}}$. The maximum of $\Delta T$ is $0.25 T$ in this range, and the SOC balancing is convergent under any load condition.

Thus, $\Delta T$ can be chosen as: 


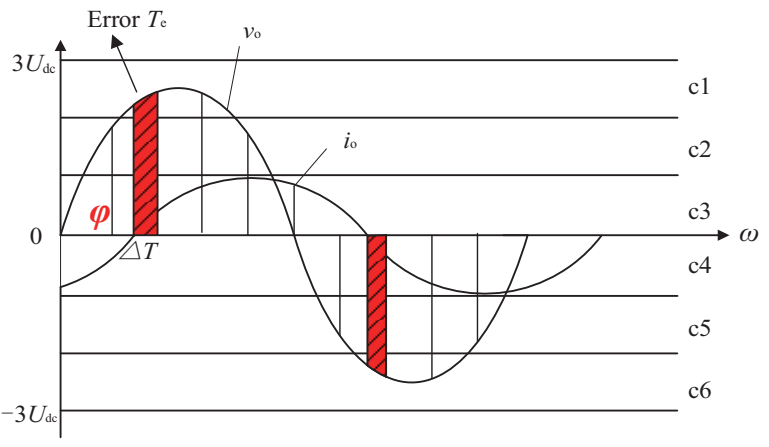

Fig. 8. Schematic diagram of SOC balancing error.

$$
\Delta T=\frac{0.5 T}{i}, i=2, \Delta T=\frac{0.5 T}{T_{\mathrm{s}}}
$$

It should be noted that the instantaneous power is used for power-matching in the proposed method. The instantaneous power at the phase angle $\varphi$ should converse. However, the powermatching is maintained within one balancing cycle $\Delta T$. Hence, the part of time that the power-matching is supposed to update is called error time of matching $T_{\mathrm{e}}$, which is shown in Fig. 8.

As shown in Fig. 8, the red part $T_{\mathrm{e}}$ needs to be small. At this point, the SOC control cycle is selected under the most common operating load of the system to achieve the fastest balancing speed.

In case of inductive load:

$$
\begin{gathered}
T_{\mathrm{e}}=\bmod \left(1-\frac{\varphi}{\omega}, \Delta T\right) \\
T_{\text {emin }}=\min \left[\bmod \left(1-\frac{\varphi}{\omega}, \Delta T\right)\right]
\end{gathered}
$$

Where mod means remainder after division.

In case of capacitive load:

$$
\begin{gathered}
T_{\mathrm{e}}=\bmod \left(\frac{|\varphi|}{\omega}, \Delta T\right) \\
T_{\text {emin }}=\min \left[\bmod \left(\frac{|\varphi|}{\omega}, \Delta T\right)\right]
\end{gathered}
$$

It should be noted that $\Delta T$ corresponding to the $T_{\text {emin }}$ is the optimal balancing cycle. If the minimum values are equal, according to the previous analysis of switching loss, the large one is taken as the optimal balancing cycle.

In order to ensure waveform output quality and avoid frequent power sorting and switching transitions. The proposed SOC balancing method in this paper employs a combination of short cycle and long cycle switching. The long balancing cycle $\Delta T$ is responsible for keeping the SOC balanced by sorting the power and distributing the modulation signal to each module. while short switching cycle $T_{\mathrm{s}}$ is utilized to shape the output voltage and current.

\section{Simulation And ExPERIMENT ANALYsis}

In order to verify the performance of the proposed balancing method based on power-matching, four case studies are developed
TABLE II

SYSTEM PARAMETERS

\begin{tabular}{lcc}
\hline \hline Symbol & Parameters & Value \\
\hline$U_{\mathrm{dc}}$ & DC side electromotive force & $70 \mathrm{~V}$ \\
$L_{\mathrm{f}}$ & Filter impedance(inductance) & $0.8 \mathrm{mH}$ \\
$C_{\mathrm{f}}$ & Filter impedance(capacitance) & $80 \mu \mathrm{F}$ \\
$U_{\text {ref }}$ & Reference voltage & $160 \mathrm{~V}$ \\
\hline \hline
\end{tabular}

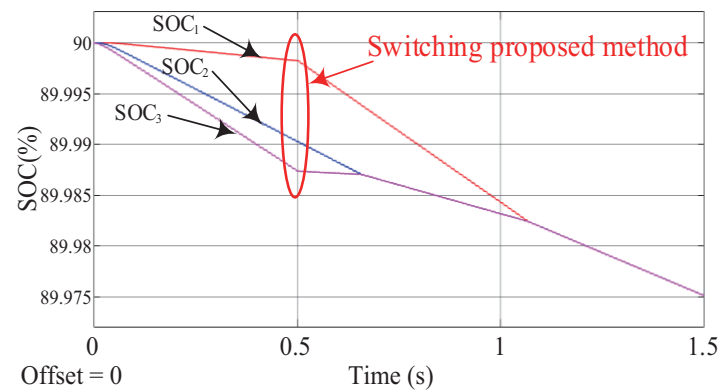

(a)

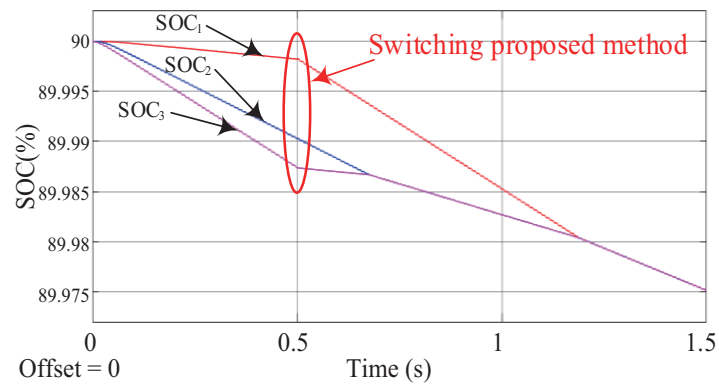

(b)

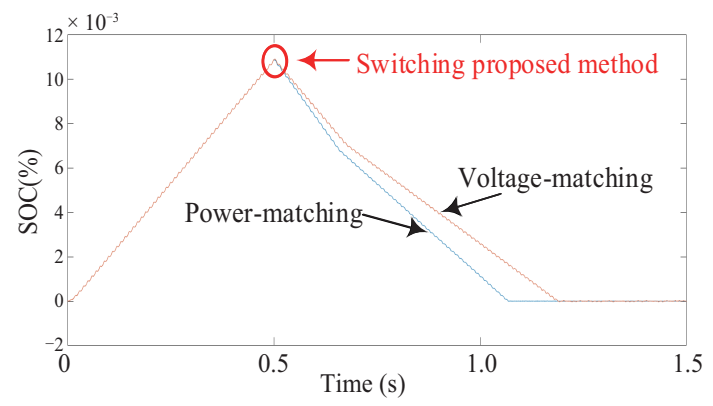

(c)

Fig. 9. SOCs of the modules before and after the switching proposed method. (a) The summary diagram of modules SOC by using power-matching. (b) The summary diagram of modules SOC by using voltage-matching. (c) SOC difference between $\mathrm{SOC}_{1}$ and $\mathrm{SOC}_{3}$.

in this section. The system parameters are detailed in Table II.

\section{A) Result for Proposed Method}

In case I, an inductive load with impedance is $(6+j 7.85) \Omega$ is employed to verify the effectiveness of the proposed method. The proposed method is enabled at $0.5 \mathrm{~s}$, and the initial values of three batteries are equal, $S O C_{1}=S O C_{2}=S O C_{3}=90 \%, \Delta T=$ $0.0002 \mathrm{~s}, T_{\mathrm{s}}=0.0002 \mathrm{~s}$.

Fig. 9(a) shows the overall power-matching based SOC balancing process of each module. Fig. 9(b) shows the overall 


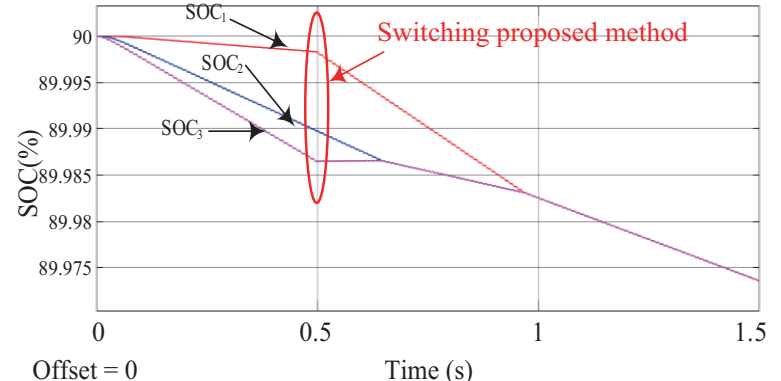

(a)

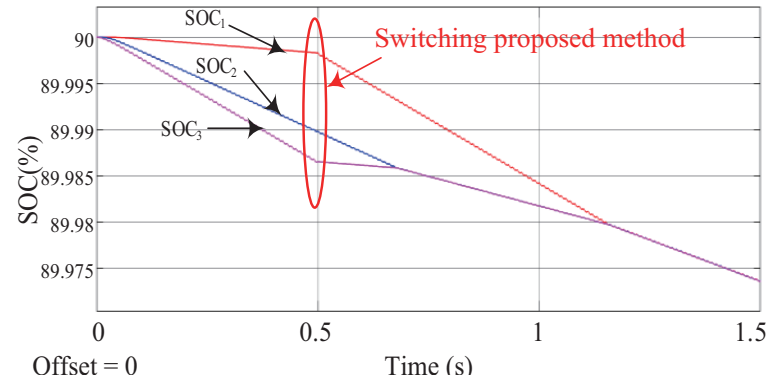

(b)

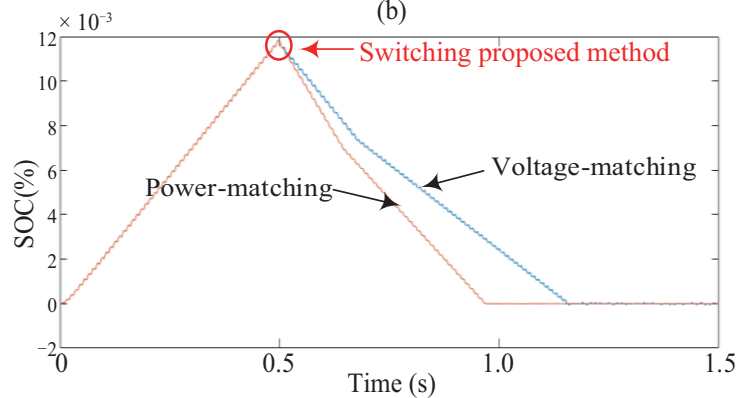

(c)

Fig. 10. SOCs of the modules before and after the switching proposed method. (a) The summary diagram of modules SOC by using power-matching. (b) The summary diagram of modules SOC by using voltage-matching. (c) SOC difference between $\mathrm{SOC}_{1}$ and $\mathrm{SOC}_{3}$.

voltage-matching based SOC balancing process of each module. Fig. 9(c) shows the SOC difference between $\mathrm{SOC}_{1}$ and $\mathrm{SOC}_{3}$. The red line is power-matching and the blue line is voltagematching. It can be seen that SOC differences gradually increase before $0.5 \mathrm{~s}$ when the proposed method is not enabled. When the proposed method works after $t=0.5 \mathrm{~s}$, the SOC differences gradually decrease to 0 . By power-matching based balancing method, the SOC of each module become balanced at $1.07 \mathrm{~s}$. By the voltage-matching based balancing method, the SOC of each module become balanced at $1.19 \mathrm{~s}$.

In case II, a capacitive load with impedance is $(8-j 7.85) \Omega$ is employed to verify the effectiveness of the proposed method. The proposed method is enabled at $0.5 \mathrm{~s}$, and the initial value of three batteries are equal, $S O C_{1}=S O C_{2}=S O C_{3}=90 \%, \Delta T=$ $0.0002 \mathrm{~s}, T_{\mathrm{s}}=0.0002 \mathrm{~s}$.

Fig. 10(a) shows the overall power-matching based SOC balancing process of each module. Fig. 10(b) shows the voltage-matching based SOC balancing process of each module. Fig. 10(c) shows the SOC difference between $\mathrm{SOC}_{1}$ and $\mathrm{SOC}_{3}$. The red line is power-matching and the blue line

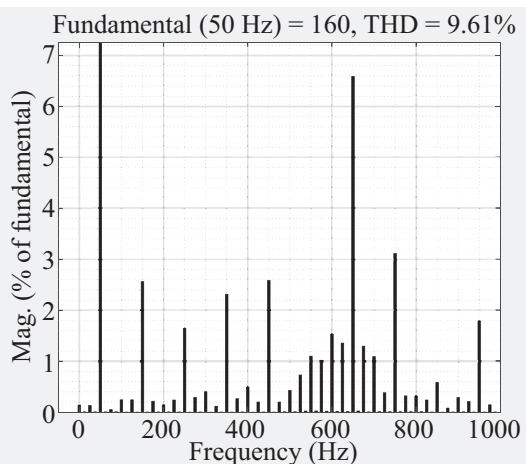

(a)

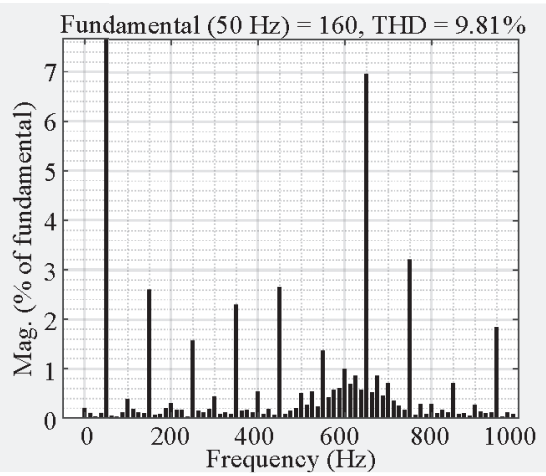

(b)

Fig. 11. The THD of output voltage under inductive load. (a) Power-matching and (b) voltage-matching.

is voltage-matching. By enabling the power-matching based balancing method at $t=0.5 \mathrm{~s}$, the SOC of each module become balanced at $0.97 \mathrm{~s}$. By the voltage-matching based balancing method, the SOC of each module become balanced at $1.19 \mathrm{~s}$.

Fig. 11 is the total harmonic distortion (THD) of output voltage under inductive load. Fig. 12 is the THD of output voltage under capacitive load. Compared by the powermatching and the voltage-matching, it can be seen that the proposed method has the same quality of output voltage.

According to the simulation, it can be found that powermatching based SOC balancing is faster than voltage-matching based SOC balancing without zero detection circuit.

\section{B. Comparison of Proposed Method with Different $\Delta T$}

In order to verify the effectiveness of the $\Delta T$ setting method proposed in this paper, different balancing cycles $(\Delta T=0.0004$, $0.0005,0.001,0.002,0.0025,0.005)$ are compared.

In case III: $T_{\mathrm{s}}=0.0002 \mathrm{~s}, S O C_{1}=90.030 \%, S O C_{2}=$ $90.025 \%, S_{3}=90.020 \%$, load impedance for $(6+j 7.85) \Omega$. The power difference and SOC difference between $\mathrm{SOC}_{1}$ and $\mathrm{SOC}_{3}$ are shown in Fig. 13.

Fig. 13 (a) is the power difference between $\mathrm{SOC}_{1}$ and $\mathrm{SOC}_{3}$. The summary of simulation data is shown in Table III case III. According to (29), it can be found that the simulation results are consistent with the theory.

$$
\begin{aligned}
& T_{\mathrm{e}(\Delta T=0.0005)}=T_{\mathrm{e}(\Delta T=0.001)}<T_{\mathrm{e}(\Delta T=0.0004)}<T_{\mathrm{e}(\Delta T=0.002)}<T_{\mathrm{e}(\Delta T=0.0025)} \\
= & T_{\mathrm{e}(\Delta T=0.005) .}
\end{aligned}
$$




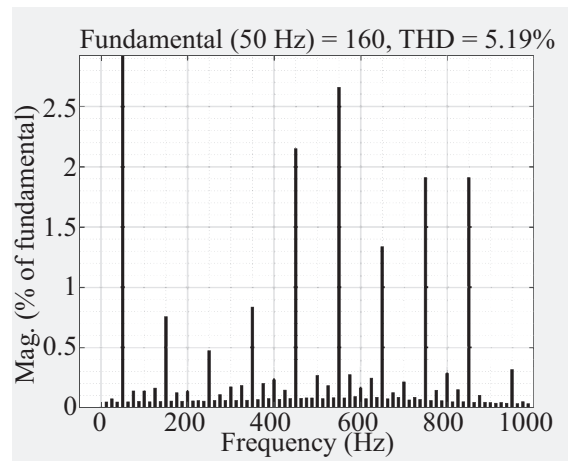

(a)

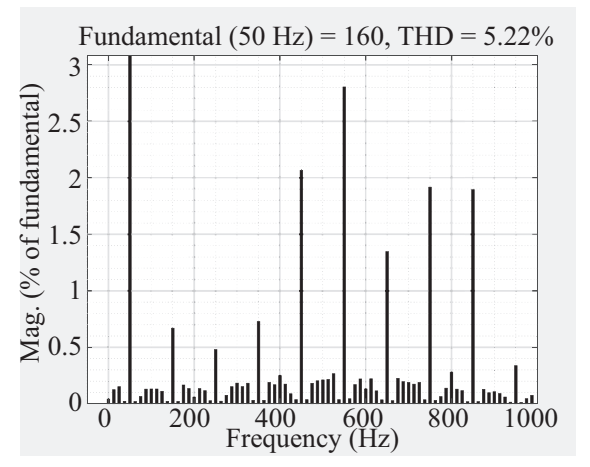

(b)

Fig. 12. The THD of output voltage under capacitive load. (a) Power-matching and (b) voltage-matching.

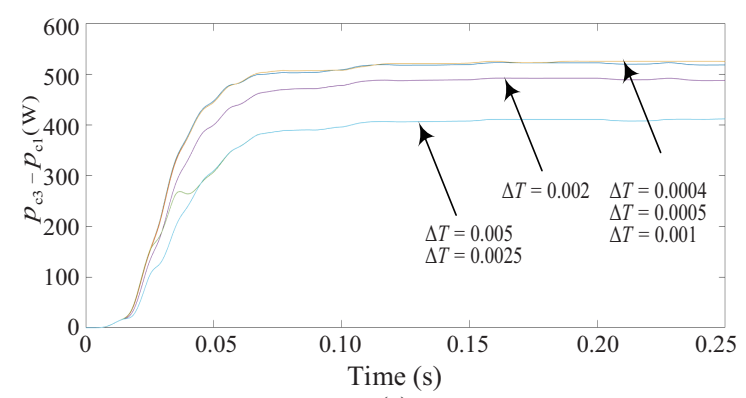

(a)

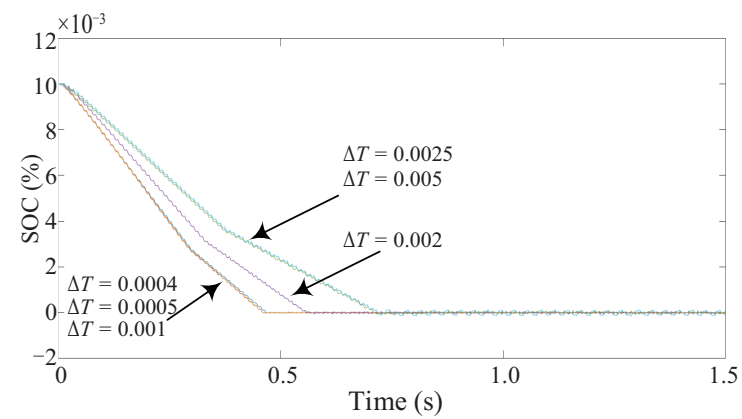

(b)

Fig. 13. The power difference and SOC difference between $\mathrm{SOC}_{1}$ and $\mathrm{SOC}_{3}$. (a) Power difference and (b) SOC difference.

Although $\Delta T=0.0005$ and $\Delta T=0.001$ balance at the same time, the switching frequency of $\Delta T=0.0005$ is higher than that of $\Delta T=0.001$. So $\Delta T=0.001$ is better.
TABLE III

SWITCHING FreQuency and BALANCING Time

\begin{tabular}{lccc}
\hline \hline $\begin{array}{l}\text { SOC balancing cycle } \\
\Delta T\end{array}$ & $\begin{array}{c}\text { Switching } \\
\text { frequency } \\
(\mathrm{Hz})\end{array}$ & $\begin{array}{c}\text { Balancing } \\
\text { time } \\
(\mathrm{s})\end{array}$ & $\begin{array}{c}\text { Power } \\
\text { difference } \\
(\mathrm{W})\end{array}$ \\
\hline & & Case III & \\
0.0004 & 6074 & 0.47 & 495 \\
0.0005 & 5864 & 0.46 & 500 \\
0.0010 & 5608 & 0.46 & 500 \\
0.0020 & 5380 & 0.56 & 450 \\
0.0025 & 5248 & 0.72 & 340 \\
0.005 & 5148 & 0.72 & 340 \\
\hline & & Case IV & \\
0.0004 & 6086 & 0.37 & 455 \\
0.0005 & 5896 & 0.37 & 455 \\
0.0010 & 5589 & 0.37 & 455 \\
0.0020 & 5342 & 0.37 & 455 \\
0.0025 & 5250 & 0.50 & 365 \\
0.005 & 5180 & 0.50 & 365 \\
\hline \hline
\end{tabular}

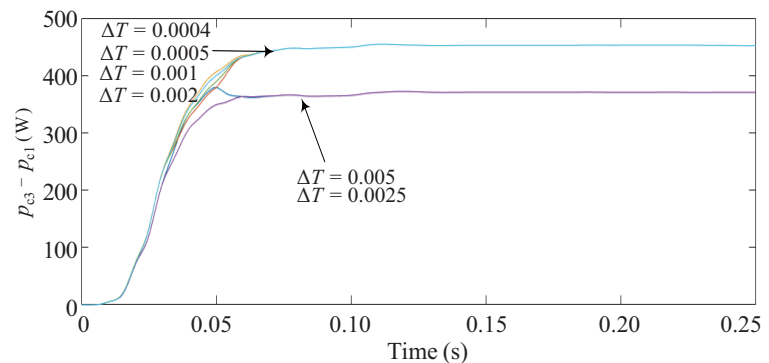

(a)

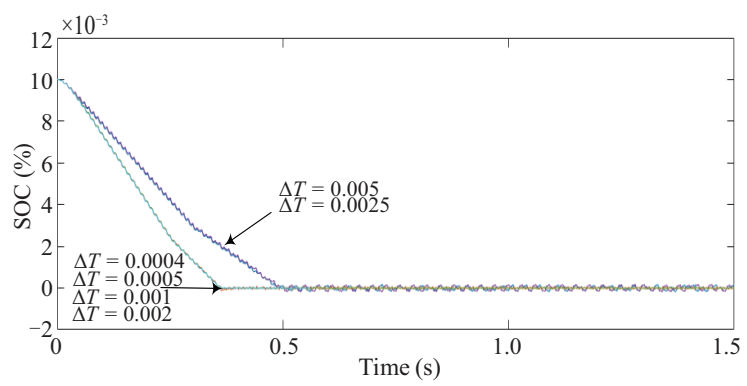

(b)

Fig. 14. The power difference and SOC difference between $\mathrm{SOC}_{1}$ and $\mathrm{SOC}_{3}$. (a) Power difference and (b) SOC difference.

In case IV: $T_{\mathrm{s}}=0.0002 \mathrm{~s}, S O C_{1}=90.030 \%, S O C_{2}=$ $90.025 \%, S_{3}=90.020 \%$, load impedance for $(8-j 7.85) \Omega$. The power difference and SOC difference between $\mathrm{SOC}_{1}$ and $\mathrm{SOC}_{3}$ are shown in Fig. 14 .

Fig. 14 (a) is the power difference between $\mathrm{SOC}_{1}$ and $\mathrm{SOC}_{3}$. The summary of simulation data is shown in Table III case IV. According to (31), it can be found that the simulation results are consistent with the theory.

$T_{\mathrm{e}(\Delta T=0.0004)}=T_{\mathrm{e}(\Delta T=0.0005)}=T_{\mathrm{e}(\Delta T=0.001)}=T_{\mathrm{e}(\Delta T=0.002)}<T_{\mathrm{e}(\Delta T=}$ ${ }_{0.0025)}=T_{\mathrm{e}(\Delta T=0.005)}$.

The optimal value of balancing cycle is $\Delta T=0.0020 \mathrm{~s}$.

According to the simulation, the following conclusions can be obtained: With the increase of $\Delta T$, the average switching frequency will decrease. In addition, the balancing speed will 


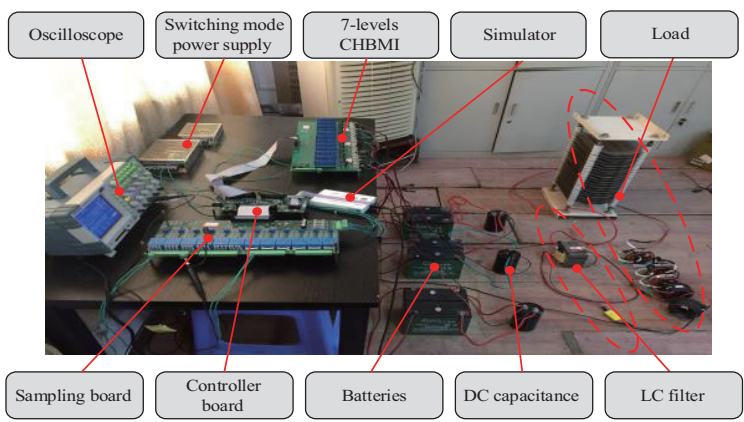

Fig. 15. The experiment platform.

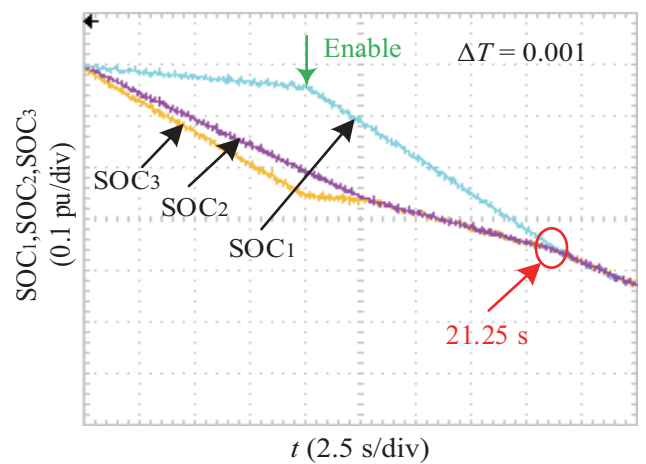

(a)

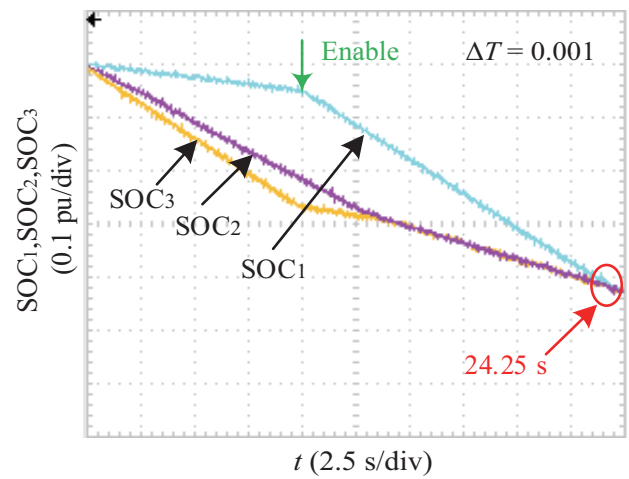

(b)

Fig. 16. The experimental results of SOC balancing. (a) Power-matching and (b) voltage-matching.

be affected by $\Delta T$. According to the law above, low switching loss and fast balancing speed have been achieved by reasonable parameters design.

\section{Experiment Results}

In order to further verify the proposed approach, the experimental test is conducted. As shown in Fig. 15, the experimental rig is set up according to the system structure in Fig. 1. The controller uses TMS320F28335, CPLD is XC9572XL and the $\mathrm{ADC}$ is $\mathrm{AD} 7580$.

\section{1)Results of Power-Matching and Voltage-Matching Under} Inductive Load

The experimental parameters are selected the same as case I. SOC initial value setting: $S O C_{1}=S O C_{2}=S O C_{3}=90 \%$. The

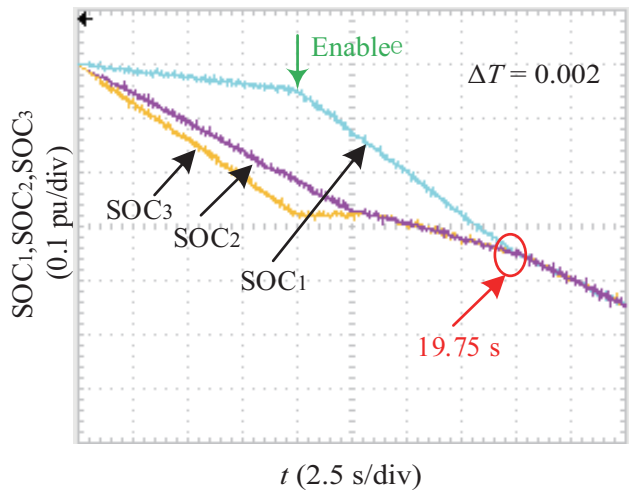

(a)

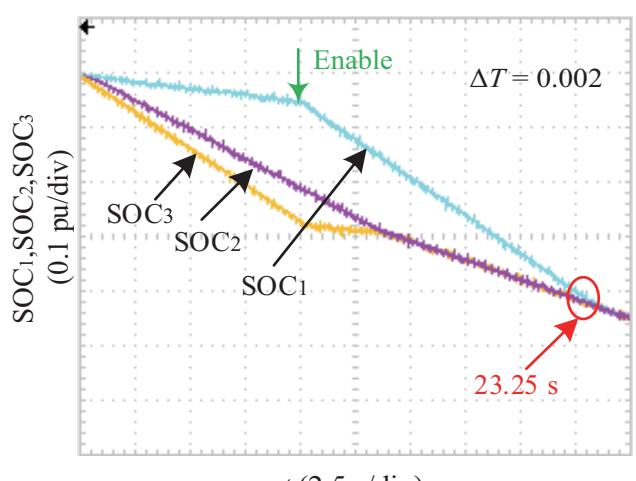

$t(2.5 \mathrm{~s} / \mathrm{div})$

(b)

Fig. 17. The experimental results of SOC balancing. (a) Power-matching and (b) voltage-matching.

balancing method is enabled at $t=10 \mathrm{~s}$. Balancing cycle $\Delta T=$ $0.001 \mathrm{~s}$.

As shown in the Fig. 16(a) and (b), the power-matching based SOC balancing method achieves SOC balancing at $21.25 \mathrm{~s}$ and voltage-matching based method achieves SOC balancing at $24.25 \mathrm{~s}$. The balancing time of the three modules is reduced from $24.25 \mathrm{~s}$ to $21.25 \mathrm{~s}$.

\section{2)Results of Power-Matching and Voltage-Matching Under Capacitive Load}

The experimental parameters are selected the same as case II. SOC initial value setting: $S O C_{1}=S O C_{2}=S O C_{3}=90 \%$. The balancing method is enabled at $t=10 \mathrm{~s}$. Balancing cycle $\Delta T=0.002 \mathrm{~s}$.

As shown in the Fig. 17(a) and (b), the power-matching based SOC balancing method achieves SOC balancing at $19.75 \mathrm{~s}$ and voltage-matching based method achieves SOC balancing at $23.25 \mathrm{~s}$. The balancing time of the three modules is reduced from $23.25 \mathrm{~s}$ to $19.75 \mathrm{~s}$.

The experimental results indicate that the individual SOCs of the CHMBI based ESS can be effectively balanced with the proposed power-matching based balancing method, and fast balancing speed is kept.

\section{Conclusion}

In this paper, a power-matching based SOC balancing method is proposed for CHBMI with PD-PWM. Compared to voltage- 
matching based SOC balancing method, this method guarantees the balancing speed without zero detection circuit. The performance of the proposed method is theoretically analyzed, and the parameter design to achieve low switching loss and fast balancing speed is given. The simulation and experimental results prove the effectiveness and correctness of the method.

\section{REFERENCES}

[1] A. Nabae, I. Takahashi, and H. Akagi, "A new neutral-point-clamped PWM inverter," in IEEE Transactions on Industry Applications, vol. IA17, no. 5, pp. 518-523, Sep. 1981.

[2] J. Pou, R. Pindado, and D. Boroyevich, "Voltage-balance limits in four-level diode-clamped converters with passive front ends," in IEEE Transactions on Industrial Electronics, vol. 52, no. 1, pp. 190-196, Feb. 2005.

[3] S. Alepuz, S. Busquets-Monge, J. Bordonau, J. Gago, D. Gonzalez, and J. Balcells, "Interfacing renewable energy sources to the utility grid using a three-level inverter," in IEEE Transactions on Industrial Electronics, vol. 53 , no. 5, pp. 1504-1511, Oct. 2006

[4] J. Rodriguez, S. Bernet, P. K. Steimer, and I. E. Lizama, "A survey on neutral-point-clamped inverters," in IEEE Transactions on Industrial Electronics, vol. 57, no. 7, pp. 2219-2230, Jul. 2010.

[5] M. Malinowski, K. Gopakumar, J. Rodriguez, and M. A. Pérez, "A Survey on cascaded multilevel inverters," in IEEE Transactions on Industrial Electronics, vol. 57, no. 7, pp. 2197-2206, Jul. 2010.

[6] D. Krug, S. Bernet, S. S. Fazel, K. Jalili, and M. Malinowski, "Comparison of 2.3-kV medium-voltage multilevel converters for industrial mediumvoltage drives," in IEEE Transactions on Industrial Electronics, vol. 54, no. 6, pp. 2979-2992, Dec. 2007

[7] M. Marchesoni, M. Mazzucchelli, and S. Tenconi, "A non conventional power converter for plasma stabilization," in Proceedings of PESC ' 88 Record, 19th Annual IEEE Power Electronics Specialists Conference, Kyoto, Japan, 1988, pp. 122-129, vol.1.

[8] J. Rodriguez, P. W. Hammond, J. Pontt, R. Musalem, P. Lezana, and M. J. Escobar, "Operation of a medium-voltage drive under faulty conditions," in IEEE Transactions on Industrial Electronics, vol. 52, no. 4, pp. 10801085, Aug. 2005

[9] M. Abdelsalam, M. Marei, S. Tennakoon, and A. Griffiths, "Capacitor voltage balancing strategy based on sub-module capacitor voltage estimation for modular multilevel converters," in CSEE Journal of Power and Energy Systems, vol. 2, no. 1, pp. 65-73, Mar. 2016.

[10] L. Zhang, Y. Zou, J. Yu, J. Qin, V. Vittal, G. G. Karady, D. Shi, and Z. Wang, "Modeling, control, and protection of modular multilevel converter-based multi-terminal HVDC systems: A review," in CSEE Journal of Power and Energy Systems, vol. 3, no. 4, pp. 340-352, Dec. 2017

[11] B. P. McGrath and D. G. Holmes, "Enhanced voltage balancing of a flying capacitor multilevel converter using phase disposition (PD) modulation," in IEEE Transactions on Power Electronics, vol. 26, no. 7, pp. 1933-1942, Jul. 2011.

[12] B. P. McGrath and D. G. Holmes, "Multicarrier PWM strategies for multilevel inverters," in IEEE Transactions on Industrial Electronics, vol. 49, no. 4, pp. 858-867, Aug. 2002.

[13] Y. Ma, X. Ma, P. Li, and X. Ren, "A modulation strategy for improving output performance of matrix converter," in CPSS Transactions on Power Electronics and Applications, vol. 4, no. 3, pp. 255-262, Sep. 2019.

[14] L. A. Tolbert, Fang Zheng Peng, T. Cunnyngham, and J. N. Chiasson, "Charge balance control schemes for cascade multilevel converter in hybrid electric vehicles," in IEEE Transactions on Industrial Electronics, vol. 49, no. 5, pp. 1058-1064, Oct. 2002.

[15] Y. F. Sun and X. B. Ruan,"Power balancing control strategy of cascade multilevel inverter," in Proceedings of the CSEE, vol. 26, no. 4, pp. 126-133, 2006.

[16] Z.Chen,Y.M.Xu,X.L.Na,J.B.Sun, "Power Balance Control and Optimization Methods With Output Voltage Rotation for Cascaded Multilevel Inverter," in Proceedings of the CSEE, vol. 38, no. 4, pp. 1132-1142, 1291, 2018.

[17] K. Bi, L. Sun, Q. An, and J. Duan, "Active SOC balancing control strategy for modular multilevel super capacitor energy storage system," in IEEE Transactions on Power Electronics, vol. 34, no. 5, pp. 4981-4992, May 2019

[18] W. Jiang, L. Huang, L. Zhang, H. Zhao, L. Wang, and W. Chen, "Control of active power exchange with auxiliary power loop in a single-phase cascaded multilevel converter-based energy storage system," in IEEE Transactions on
Power Electronics, vol. 32, no. 2, pp. 1518-1532, Feb. 2017.

[19] Sara Mensou, Ahmed Essadki, Tamou Nasser, and Badr Bououlid Idrissi. "A direct power control of a DFIG based WECS during symmetrical voltage dips," in Protection and Control of Modern Power Systems, vol. 5, no. 1, pp. 36-47, 2020.

[20] P. Sochor and H. Akagi, "Theoretical and experimental comparison between phase-shifted PWM and level-shifted PWM in a modular multilevel SDBC inverter for utility-scale photovoltaic applications," in IEEE Transactions on Industry Applications, vol. 53, no. 5, pp. 46954707, Sep.-Oct. 2017.

[21] E. Chatzinikolaou and D. J. Rogers, "Cell SOC balancing using a cascaded full-bridge multilevel converter in battery energy storage systems," in IEEE Transactions on Industrial Electronics, vol. 63, no. 9, pp. 5394-5402, Sep. 2016.

[22] A. Gholizad and M. Farsadi, "A novel state-of-charge balancing method using improved staircase modulation of multilevel inverters," in IEEE Transactions on Industrial Electronics, vol. 63, no. 10, pp. 6107-6114, Oct. 2016.

[23] J. Mei, K. Shen, B. Xiao, L. M. Tolbert, and J. Zheng, "A new selective loop bias mapping phase disposition PWM with dynamic voltage balance capability for modular multilevel converter," in IEEE Transactions on Industrial Electronics, vol. 61, no. 2, pp. 798-807, Feb. 2014.

[24] K. Wang, Y. Deng, W. Li, H. Peng, G. Chen, and X. He, "Fundamental frequency sorting strategy for capacitor voltage balance of modular multilevel converters with phase disposition PWM," in Proceedings of 2016 IEEE Applied Power Electronics Conference and Exposition (APEC), Long Beach, CA, 2016, pp. 1450-1455.

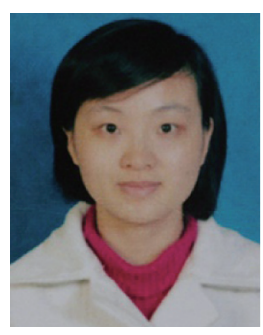

Jingrong Yu was born in Dandong, China, in 1981. She received the B.E. and Ph.D. degrees in control science and engineering from Hunan Univesity, Changsha, China, in 2004 and 2009, respectively. From 2014 to 2015, she was a Visiting Scholar with the Department of Electrical Engineering and Computer Science, The University of Tennessee, Knoxville. In 2009, she joined Central South University, where she is currently an Associate Professor. Her research interests include modeling and control of power electronic converters in renewable energy systems and microgrids, and analysis and control of power quality.

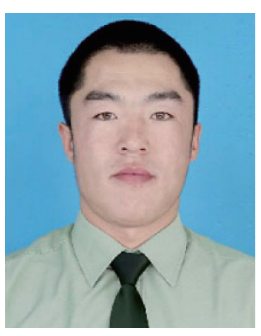

Gang Zhang was born in Ordos, China, in 1995. He received the B.S. degree in electrical engineering and automation from Central South University, Hunan, China, in 2020. He is currently pursuing the M.S. degree in electrical engineering at Central South University. His main research interests include fault diagnosis, fault tolerance and power electronics.

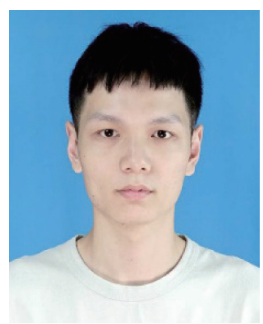

Mingkai Peng was born in Hunan, China, in 1995. He received the B.S. degree in electrical engineering and automation from Nanjing Normal University, Jiangsu, China, in 2017. He received the M.S. degree in electrical engineering from Central South University, Hunan, China, in 2020. His research interests include multilevel converters and multilevel modulations in renewable energy systems and microgrids. 


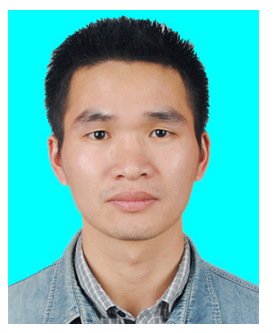

Dongran Song received the Ph.D. degree from the School of Information Science and Engineering, Central South University, Changsha, China, in 2016, where he has been an Associate Professor, since 2018. He was a Senior Engineer with China Ming Yang, Zhongshan, from 2009 to 2017, where he took part in designing 1.5-6.0-MW series wind turbines.

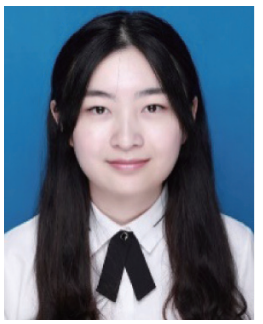

Maoyun Liu was born in Chongqing, China, in 1994. She received the B.S. degree in control science and engineering from Shandong University, Shandong, China, in 2016. She received the M.S. degree in electrical engineering from Central South University, Hunan, China, in 2019. Her research interests include modeling and control of power electronic converters in renewable energy systems and microgrids. 